\title{
COMMUNICATION NETWORK IN MIMOSA PUDICA
}

\section{S. P. DHIR}

Former Medical Superintendent and Professor, Govt. Medical College and Hospital, Chandigarh, India

Demonstration and mapping of communication networks in Mimosa Pudica Plant have important implications for Neuro-botany. For the first time, it is proposed that loss of electric charge is utilized as a signaling mechanism in Mimosa pudica. It runs contrary to the popular theory of generation of action potential giving rise to rapid drooping of leaves and folding of leaflets. Loss of electric charge leads to opening of hundreds of ion channels giving rise to rapid drooping of leaves and folding of leaflets. It is fundamentally different than the use of action potential in the animal kingdom. Present study constructs communication network in the plant using various stimuli. A rapid, widespread network of communication has been observed.
\end{abstract}

KEYWORDS: Electric Charge, Mimosa Pudica, Communication Network \& Phospholipids

Received: Dec 11, 2017; Accepted: Dec 30, 2017; Published: Jan 10, 2018; Paper Id.: IJBRFEB20181

\section{INTRODUCTION}

Mimosa pudica (Touch me not) is a creeping annual or perennial herb of the pea family Fabaceae often grown for its curiosity value. The compound leaves fold inward and droop when touched or shaken and re-open a few minutes later [1]. The leaves close rapidly in response to stimuli, such as touching, warming, blowing, or shaking. These types of movements have been termed seismonastic movements.

Volkov et al [2,3] after extensive study came to the conclusion that the movements in Mimosa pudica, such as the response to touch, appear to be regulated by electrical, hydro dynamical and chemical signal transduction.

Dhir et al [6] found acetone and other lipid solvents could also cause rapid drooping and folding of the leaves of Mimosa pudica. They proposed that lipid solvents disrupt phospholipids, which are insulating material between two charged surfaces. In contrast to generation of action potential for signal transduction, mimosa pudica (touch me not) utilizes loss of electric charge for signal transduction. Loss of electric charge as a signaling mechanism in Mimosa pudica plant is reported for the first time. It is fundamentally different than the use of action potential in the animal kingdom. Both systems have evolved for a common theme to ward off from harm. Present study traced communication network in the plant using various stimuli.

\section{MATERIALS AND METHODS}

One to two month old well watered plants were used for the study. The effect of various stimuli was studied

- Touch: Tooth pick was used to touch the areas of interest. 
- Acetone: A drop of the chemical was gently placed at the sight.

- Lipid Solvents: The cuticle was scrapped from $2,0 \mathrm{~cm}$. long stem by scrapping with blunt knife. The nude portion of stem was wrapped in cotton and charged with $1.0 \mathrm{ml}$. of lipid solvent.

- Heat: $2.0 \mathrm{~cm}$ long stem of the plant was wrapped in silver wire and temperature was raised to 65-70 degree centigrade for two minute.

Circuit diagrams were prepared by connecting the sensitive points (which caused rapid drooping of leaves and folding of leaflets) with affected parts.

\section{OBSERVATIONS}

Motor cells responsible for the drooping of the leaf and folding of the leaflets are situated at the pulvinus and petiole respectively. In general, pulvinus was more sensitive and first to be affected than petiole.

\section{Touch}

It was found that both pulvinus and petiole were sensitive to touch resulting in rapid drooping of the leaf and folding of the leaflets respectively. Petiole which are situated in two rows at the base of each leaflet respond in vertical fashion and are not connected horizontally. However the two rows are connected through the base of the leaf where charge junction is situated (Figure 1) No effect was seen on touching the stem, leafstalk or leaflets. The pulvinus and charge junction of petiole is further connected with communication lines via the leafstalk though the leafstalk itself is not sensitive to touch (Figure 2)

\section{Acetone}

Application of acetone had no effect on the stem. Application of acetone drop to pulvinus, leafstalk, charge junction and petiole caused a rapid drooping of the leaf at pulvinus and folding of leaflets respectively Application of acetone to the junction of leafstalk with leaflets led to drooping of pulvinus as well as folding of leaflets. Application of acetone to petiole led to folding of the leaflets. If the stimulus was applied to the middle of the leaflet the leaflets folded both in upward and downward direction. Application of acetone to leaflets had no effect on the leaflets. Affected parts namely, pulvinus and petiole recovered in 10-15 minutes.

Acetone and other lipid solvents when applied to stem after the scrapping of the cuticle showed widespread though unpredictable effects. Many a times such application produced earliest response in another shoot starting at the top ( Figure 3.)

\section{Heat}

Application of heat to the stem of the plant at various locations, namely at the bottom and other places resulted in rapid dropping of the leaf and folding of the leaflets. The stem was otherwise insensitive to touch and acetone. Affected parts, namely pulvinus and petiole recovered in 15-20 minutes. Sometimes the leaves of other shoots were also affected. It is observed that:

- There is a definite communication link between each petiole in a vertical fashion and not horizontally. Further petiole are connected to pulvinus via the leafstalk as demonstrated by touch stimulus. 
- There is a common communication link between pulvinus, leafstalk and petiole as demonstrated by acetone as stimulus.

- There is a communication link between stem, pulvinus, leafstalk and petiole as demonstrated by application of heat to the stem of the plant.

- The communication network was not restricted to the stem- leaves but also to other shoots of the plant.

\section{DISCUSSIONS}

The study demonstrates a rapid wide network of communication in the mimosa pudica plant. Loss of electric charge has been suggested by Dhir et al [4,5] for the transmission of stimulus as well as loss of turgor of the motor cells in bottle gourd plant tendril. In Mimosa there are hundreds of ion channels which open up due to loss of electric charge and rapid loss of turgor of motor cells. It is proposed that there is a charged surface (lamina) which runs between petiole, leafstalk, pulvinus and stem. This lamina acts like a conductor of an electric charge. These lamina join other lamina like capacitors rather than continuous electric conductor. Loss of charge in one lamina may lead to loss of charge on another lamina because of lack of lateral charge support or capacitor connections.

The charged lamina is separated from the other conductive material by phospholipids which act as insulation. Loss of electric charge can be due to shortening of two surfaces by touch as happens in bottle gourd tendril [4,5] and touch phones. Disruption of phospholipids by acetone or heat can also lead to loss of charge and loss of turgor of motor cells. Phospholipids reorganize themselves providing insulation and recharging of the lamina and recovery of turgor. The whole process behaves like a Varistor where there is a sudden drop of resistance in the presence of harmful high voltage and self resetting. Mimosa has evolved this process of drooping and folding of leaves to protect from herbivory and self resetting. The choice of loss of charge as a means of communication is intriguing. Off effect is greater than on effect in Electrophysiology.

\section{CONCLUSIONS}

The whole system is akin to reflex action in the animal kingdom and has implications for Neuro-botany. In mimosa the insult can produce response at a distant place without analysis of the stimulus as happens in reflex action. Both responses are of protective nature. Further drooping of leaves first at the top of the plant irrespective of the site of stimulus points to some selectivity in response. Top of the plant is most vulnerable to herbivory.

Possibly a similar process takes place in concussion injuries of the brain in humans. A shake up of the brain leads to loss of insulation in neural tissue and temporary loss of function. When insulation is restored neural function is restored.

The theory of loss of charge proposed above runs contrary to the popular theory of generation of action potential[7] giving rise to rapid drooping of leaves and folding of leaflets in Mimosa pudica.

Acknowledgements are due to Mr. Ravi Kumar for technical assistance.

\section{REFERENCES}

1. Raven, Peter H.; Evert, Ray F.; Eichhorn, Susan E. (January 2005). "Section 6. Physiology of Seed Plants: 29. Plant Nutrition and Soils". Biology of Plants (7th ed.). New York: W. H. Freeman and Company. p. 639. 
2. Alexander G Volkov, Justin C Foster, Kara D Baker, Vladislav S Markin. Mechanical and electrical anisotropy in Mimosa pudica pulviniPlant Signal Behav. 2010 Oct; 5(10): 1211-1221.

3. Alexander G Volkov,Justin C Foster, Vladislav S Markin. Signal transduction in Mimosa pudica: biologically closed electrical circuits. Plant Cell Environ. 2010 May;33(5):816-27.

4. Dhir S P. Electric Charge Signaling in Plant Tendrils. International Journal of Scientific and Research Publications.2016 August; 6(8):137-8.

5. Dhir S P. Bottle Gourd Plant Tendril-Role of Electric Charge in Rapid Contact Coiling. International Journal of Scientific and Research Publications.2017 November; 7(11):447-8.

6. Dhir S P, Salunke D, Kaur A and Dhir S. Mystery of Mimosa pudica. International Journal of Botany and Research (IJBR).2017 December. 7 (6): 41-44

7. Vanden Driessche, Thérèse. "Nutations in Shoots and in Desmodium Lateral Leaflets, Nyctinastism and Seismonastism in Mimosa pudica. Comparison and Evolution of Morphology and Mechanism". Biological Rhythm Research.2000. 31 (4): 451468.

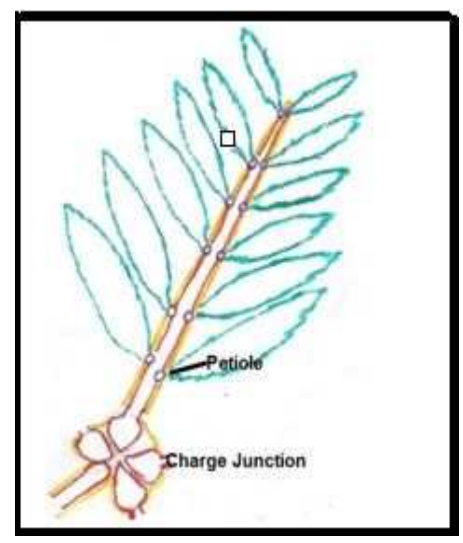

Figure 1: Schematic Diagram of the Leaf Showing Senso-Motor Cells

(Blue Circles) of Petiole Connecting each Other (Red Lines) and to the Electric Charge Junction of Four Leaves

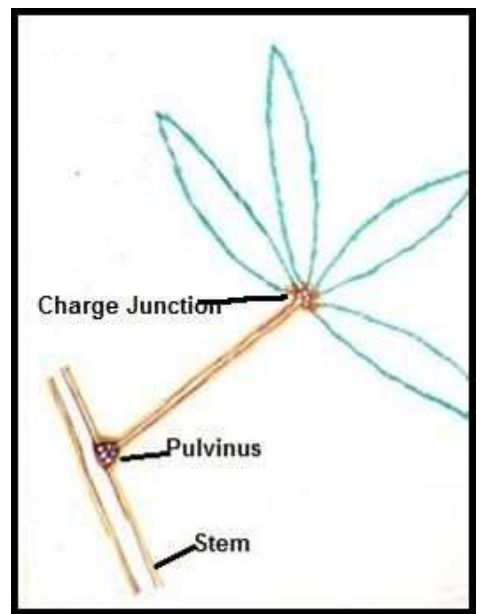

Figure 2: Schematic Diagram Showing Electric G Charge

Carrying Lamina Connecting Pulvinus and Petiole via Leaf-Stalk

(Red Lines). Blue Circles Depict Senso-Motor Cells of Pulvinus 


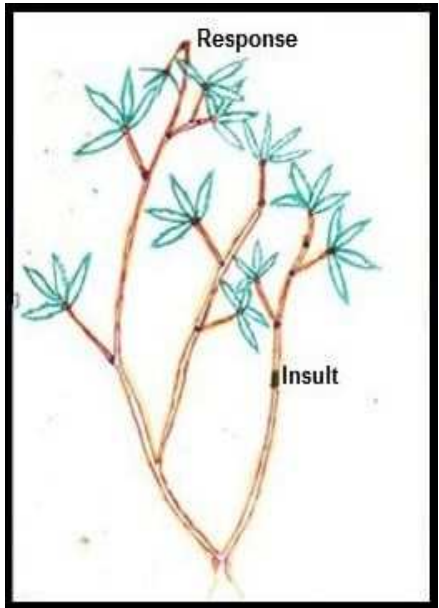

Figure3: Schematic Diagram Showing Insult to Stem at One Location and Earliest Response 
